BROWN-HET-932, hep-th/9401134

\title{
High Energy Scattering in 2+1 QCD
}

\author{
Miao Li ${ }^{1}$ and Chung-I Tan日 \\ Department of Physics \\ Brown University \\ Providence, RI 02912
}

\begin{abstract}
High energy scattering in 2+1 QCD is studied using the recent approach of Verlinde and Verlinde. We calculate the color singlet part of the quark-quark scattering exactly within this approach, and discuss some physical implication of this result. We also demonstrate, by two independent methods, that reggeization fails for the color octet channel. We briefly comment on the problem in $3+1$ QCD.
\end{abstract}

$1 / 94$

1 E-mail: li@het.brown.edu

2 E-mail: tan@het.brown.edu 


\section{Introduction}

Quantum chromodynamics (QCD) with massless quarks is classically scale invariant; it contains no explicit small parameters. Quantum mechanically, the character of QCD changes depending on the nature of available probe. At short distances, due to asymptotic freedom, the basic degrees of freedom are weakly coupled quarks and gluons. Collisions where all invariants $\left\{p_{i} \cdot p_{j}\right\}$ are large probe short-distance physics and can be treated perturbatively. Over the past two decades, perturbative tests of QCD have been met with continued success, leaving little doubt on it being the correct theory of strong interactions. As one moves to larger distance scales, the coupling strength increases and one enters the nonperturbative quark-gluon confinement regime. Short of resulting to lattice numerical studies, the most promising tool for a non-perturbative treatment of QCD which builds in confinement naturally remains the topological treatment based on $1 / N_{c}$ expansion. Indeed, many qualitative features of low energy hadronic phenomenology can best be understood in such a setting. Unfortunately, quantitative calculational scheme is still lacking, and it is unlikely one could be developed in the near future.

Formally, a high energy hadronic collision in the near-forward limit corresponds to the mixing of a "short-distance" phenomenon in the longitudinal coordinates with a "longdistance" phenomenon in the transverse coordinates. By treating the longitudinal and transverse degrees of freedom separately, one could hope that a "dimensional reduction" scheme can be formulated, reducing QCD at high energies to an effective two-dimensional field theory. An interesting attempt in this direction has been made recently by Verlinde and Verlinde [1]. The effective theory involves several fields and two coupling constants, the original gauge coupling $g$ and an effective coupling $e^{2} \sim g^{2} \log s$.

In this paper, we would like to explore the viability of this approach further by study a simpler situation, high energy scattering of QCD in $2+1$ dimensions, where the resulting effective theory is one-dimensional.

In Ref. [1], one first accepts the conventional wisdom that the high energy behavior of hadron-hadron near-forward amplitudes in QCD can be extracted by studying the corresponding quark-quark scattering amplitudes. One further assumes that the latter can be expressed in terms of correlators of certain Wilson lines [2]. Denote the center of mass squared by $s$ and the momentum transfer squared by $t$; one is interested in the region

where $s>|t|$. One normally also assumes that $|t|$ is still greater than $\Lambda_{Q C D}$. This later condition hopefully ensures that the perturbation makes sense. As we shall see, Verlindes' 
formulation presumes no perturbative expansion, and indeed explicit nonperturbative calculation can actually be done in this approach when one considers $2+1$ QCD. For related approaches, see [3].

Let $\alpha$ be the longitudinal index and $i$ the transverse index. Starting with the standard Yang-Mills action, let us scale the longitudinal coordinates by a factor $\lambda=1 / \sqrt{s}$, which corresponds to a scaling of longitudinal momenta by $\sqrt{s}$. It follows that components $F_{\alpha \beta}$ of the field strength are scaled by a factor $\lambda^{-2}, F_{\alpha i}$ by a factor $\lambda^{-1}$, with $F_{i j}$ left intact, i.e., the Yang-Mills action now reads as

$$
S=\frac{1}{4 g^{2}} \int d^{4} x \operatorname{tr}\left(\lambda^{-2} F_{\alpha \beta}^{2}+2 F_{\alpha i}^{2}+\lambda^{2} F_{i j}^{2}\right)
$$

Verlinde and Verlinde first assume that, with $\lambda$ small at high energies, one can neglect the third term in the above action. We note here that in working with $2+1$ dimensions, this term is actually absent, because there is only one transverse dimension. It thus removes one possible sources of uncertainties concerning this novel approach [4].

The next crucial assumption made in [1] is that the fluctuation of the longitudinal components is suppressed due to the first term in the above equation. $A_{\alpha}$ is therefore flat in the longitudinal directions and one can replace it by $\partial_{\alpha} G G^{-1}$ in the second term. Finally, one integrates over longitudinal coordinates, leading to an effective two dimensional action, involving seven matrix fields. Unfortunately, this effective theory is still too complicated to be amenable to explicit calculation. As we shall see, by studying $2+1$ QCD, our effective theory is one-dimensional, involving only three independent fields. This allows us to study the model both perturbatively as well as exactly in $e^{2}(s)$.

In order to provide some relevant background for proper appreciation of the possible significance of the approach of Ref. [1], we recall that one of the most striking aspects of high-energy hadron-hadron scattering is the continued increase of the total cross section $\sigma_{T}$ with the energy. Traditional approach to high energy near-forward hadronic collisions invariably involves the notion of Pomeron. A rising cross section first requires a Pomeron with a zero momentum transfer intercept greater than one, which, if uncorrected, would lead to the violation of Froissart bound at asymptotic energies. Either through an eikonal or other more elaborate schemes, screening corrections hopefully would then lead to an expanding disk picture.

There are currently two seemingly conflicting interpretations of Pomeron in QCD: One based on perturbative leading log approximation (LLA) [5] [6] [7] [8] and another 
based on nonperturbative (large- $N$ and/or phenomenological) consideration [9] [10]. In a perturbative treatment, a Pomeron loosely corresponds to the color-singlet bound state of two (reggeized) gluons. In a nonperturbative treatment, the Pomeron is thought to correspond to nonperturbative gluon exchanges having the topology of a closed string.

One potential advantage of the Verlindes' approach, as stated earlier, is the fact that it offers the possibility of providing either an alternative perturbative LLA treatment or a starting point for nonperturbative studies. An attempt in unifying both perturbative and nonperturbative aspects of high energy hadron collisions has been made in [11]. In generating a perturbative Pomeron [5] [8], it is important to identify on the one hand the "Lipatov vertex" and on the other hand the reggeization of gluons. The former has been demonstrated in Ref.[1], but the possibility of gluon reggeization remains unclear. In section 3 we demonstrate that, under Verlindes' approach, reggeization does not occur for $2+1$ QCD at high energies. This may pose a question as whether one need modify the ansatz for how $\log s$ enters the effective action.

Although the above result might cast doubts on the reliability of the Verlindes' scheme, it is nevertheless interesting to push the program further for extracting other nonperturbative consequences. We begin first by introducing a new procedure in section 4 which allows to perform nonperturbative analysis. In section 5 we discuss exact result for the quark-quark high energy scattering for $2+1 \mathrm{QCD}$ under Verlindes' treatment. In section 6 we discuss hadron-hadron scattering in $2+1 \mathrm{QCD}$ and briefly comment on the case of $3+1$ QCD.

\section{Verlindes' approach to high energy scattering in QCD}

In this section we first briefly review Verlindes' approach [1] to high energy quarkquark scattering at fixed momentum transfer. We then simplify their effective action to set the framework for calculation of scattering amplitude.

Consider quark-quark scattering in QCD with center of mass energy squared $s . s$ is very large, and the momentum transfer $t$ is much smaller than $s$, but still greater than $\Lambda_{Q C D}$. This later condition ensures that the perturbation makes sense. Indeed, as we shall see, Verlindes' formulation presumes no perturbative expansion, and indeed nonperturbative calculation can be done in this formulation when one considers $2+1$ QCD. 
Under the same assumption where one drops lower order terms under the longitudinal scale transformation, the quark-quark scattering amplitude can be expressed in terms of correlation of two Wilson lines

$$
A\left(s, t=-q^{2}\right)=-\frac{i s}{2 m_{q}^{2}} \int d^{2} z e^{-i q z}\left\langle\left(V_{+}(0)-1\right)\left(V_{-}(z)-1\right)\right\rangle,
$$

where $V_{ \pm}=e^{\int_{-\infty}^{\infty} d x^{ \pm} A_{ \pm}}$, involve longitudinal gauge field components only. The pre-factor $\frac{i s}{2 m_{q}^{2}}$ comes from kinematics in longitudinal dimensions, $m_{q}$ is the quark mass (we assume the two quarks have the same mass, although this is not necessary). A detailed justification of the use of Wilson lines for quark-quark scattering can be found in Ref. [2].

As stated in the introduction, we accept that it is justifiable at high energies to approximate $A_{\alpha}$ by the pure gauge condition $\partial_{\alpha} G G^{-1}$ while dropping the first term $\lambda^{-2} F_{\alpha \beta}^{2}$ in Eq.(1.1). One next integrates over longitudinal coordinates, assuming that the gauge transformed $A_{i}$ satisfies classical equation $\partial_{+} \partial_{-}\left(G^{-1} D_{i} G\right)=0$. An effective two dimensional action is thus obtained, involving fields at four end-points of two quark trajectories. There are totally six fields in this action. $g_{A}, A=1,2$, are values of $G$ at the two ends of the left moving quark. $h_{A}, A=1,2$, are values of $G$ at the two ends of the right moving quark. $a_{i}^{ \pm}$are gauge fields associated with the left moving and the right moving trajectories respectively.

This effective action is singular in that some fields have a singular propagator. Verlindes then regularize the action by noting the fact that both quarks are actually not exactly light-like. Their classical trajectories depart from their light-cones by an amount proportional to $1 / \sqrt{s}$. The propagator of transverse components $A_{i}$ then acquires $s$ dependence through logs. We shall not go through their derivation, but just quote their result. The regularized action is

$$
S\left[g_{A}, h_{B}, a_{i}^{ \pm}\right]=\frac{1}{g^{2}} \int d^{2} z M^{A B} \operatorname{tr}\left(g_{A}^{-1} D_{i}^{+} g_{A} h_{B}^{-1} D_{i}^{-} h_{B}\right),
$$

where the matrix $M$ is

$$
\begin{aligned}
M & =\left(\begin{array}{cc}
1+\epsilon & -1+\epsilon \\
-1+\epsilon & 1+\epsilon
\end{array}\right) \\
\epsilon^{-1} & =1-\frac{2 i}{\pi} \log s,
\end{aligned}
$$

and covariant derivatives are given by $D_{i}^{+} g_{A}=\left(\partial_{i}+a_{i}^{+}\right) g_{A}, D_{i}^{-} h_{A}=\left(\partial_{i}+a_{i}^{-}\right) h_{A}$. Here a comment about the coefficient $1 / g^{2}$ in the action (2.2) is in order. We shall compare 
result of perturbative calculations based on (2.2) to the conventional perturbation results, so we need be careful in setting the same convention about the coupling constant. From the rescaled action (1.1), the weighting coefficient of the second term is $1 /\left(2 g^{2}\right)$. A factor 2 comes from writing the second term in the light-cone coordinates and integrating out the longitudinal coordinates.

The Wilson lines operators $V_{ \pm}$introduced earlier for calculating the quark-quark scattering amplitude can be written in terms of $g_{A}$ and $h_{A}$, since $A_{ \pm}=\frac{1}{g} \partial_{ \pm} G G^{-1}$. Let $g=g_{2} g_{1}^{-1}$ and $h=h_{2} h_{1}^{-1}$, then $V_{+}=g, V_{-}=h$. The quark-quark amplitude then becomes

$$
A\left(s, t=-q^{2}\right)=-\frac{i s}{2 m_{q}^{2}}\langle(g-1)(q)(h-1)(-q)\rangle .
$$

We shall next simplify the effective action (2.2) before discussing the correlation of these two Wilson line operators.

So far we have not fixed any gauge. Consider the $a^{ \pm}$independent part of (2.2) first:

$$
\begin{aligned}
S(g, h) & =\frac{1}{g^{2}} \int d^{2} z\left[(1+\epsilon) \operatorname{tr}\left(g_{2}^{-1} \partial_{i} g_{2}-g_{1}^{-1} \partial_{i} g_{1}\right)\left(h_{2}^{-1} \partial_{i} h_{2}-h_{1}^{-1} \partial_{i} h_{1}\right)\right. \\
& +2 \epsilon \operatorname{tr}\left[g^{-1} \partial_{i} g_{1}\left(h_{2}^{-1} \partial_{i} h_{2}-h_{1}^{-1} \partial_{i} h_{1}\right)+h_{1}^{-1} \partial_{i} h_{1}\left(g_{2}^{-1} \partial_{i} g_{2}-g_{1}^{-1} \partial_{i} g_{1}\right)\right] \\
& \left.+4 \epsilon \operatorname{tr}\left(g_{1}^{-1} \partial_{i} g_{1} h_{1}^{-1} \partial_{i} h_{1}\right)\right] .
\end{aligned}
$$

It is easy to see from the above equation that fluctuations of $g_{2}^{-1} \partial_{i} g_{2}-g_{1}^{-1} \partial_{i} g_{1}$ and $h_{2}^{-1} \partial_{i} h_{2}-h_{1}^{-1} \partial_{i} h_{1}$ are controlled by $g^{2}$, and fluctuations of $g_{1}^{-1} \partial_{i} g_{1}$ and $h_{1}^{-1} \partial_{i} h_{1}$ are controlled by $g^{2} \epsilon^{-1}$, a parameter much greater than $g^{2}$. Therefore, the second term in the above action is negligible compared to the first term and the third term. Next, observe that

$$
4 \epsilon \operatorname{tr}\left(g_{1}^{-1} \partial_{i} g_{1} h_{1}^{-1} \partial_{i} h_{1}\right)=\epsilon \operatorname{tr}\left(\left(g_{1}^{-1} \partial_{i} g_{1}+h^{-1} \partial_{i} h_{1}\right)^{2}-\left(g_{1}^{-1} \partial_{i} g_{1}-h_{1}^{-1} \partial_{i} h_{1}\right)^{2}\right),
$$

and $g_{1}^{-1} \partial_{i} g_{1}+h_{1}^{-1} \partial_{i} h_{1}$ essentially decouples from the action, if we ignore the second term in the action. Now recalling the definition $g=g_{2} g_{1}^{-1}, h=h_{2} h_{1}^{-1}$ and defining $G=g_{1} h_{1}^{-1}$, the $a^{ \pm}$independent part of the effective action is simplified to

$$
S[g, h, G]=\frac{1}{g^{2}} \int d^{2} z\left[\operatorname{tr}\left(g^{-1} \partial_{i} g G h^{-1} \partial_{i} h G^{-1}\right)-\epsilon \operatorname{tr}\left(G^{-1} \partial_{i} G\right)^{2}\right],
$$

where we have ignored the term proportional to $\epsilon$ in the first term in (2.5). It is seen that this action is written in terms of $g, h$, "physical" fields associated with Wilson lines, and $G$ 
which plays an important role as a coupling field. In $2+1$ QCD, (2.5) is the whole effective action, if we choose the Landau gauge $a^{ \pm}=0$.

In $3+1 \mathrm{QCD}$, we can not choose a gauge in which $a_{i}^{ \pm}=0$. There are two parts in the $a^{ \pm}$-dependent part of the effective action. The first part contains no $\epsilon$, and can be neatly included in (2.5) by replacing $g^{-1} \partial_{i} g$ and $h^{-1} \partial_{i} h$ with $g^{-1} D_{i}^{+} g$ and $h^{-1} D_{i}^{+} h$ respectively. Here we define $D_{i}^{+} g=\partial_{i} g+\left[a_{i}^{+}, g\right]$ and $D_{i}^{-} h=\partial_{i} h+\left[a_{i}^{-}, h\right]$. The second part is proportional to $\epsilon$. Once again, we can drop out some terms in this part and keep the most relevant one, which is

$$
\frac{1}{g^{2}} \int d^{2} z 4 \epsilon \operatorname{tr}\left(a_{i}^{+} G a_{i}^{-} G^{-1}\right)
$$

Finally, we add the $a^{ \pm}$-dependent part to (2.5) and obtain the full effective action

$$
S\left[g, h, G, a^{ \pm}\right]=\frac{1}{g^{2}} \int d^{2} z \operatorname{tr}\left[g^{-1} D_{i}^{+} g G h^{-1} D_{i}^{-} h G^{-1}-\epsilon\left(G^{-1} \partial_{i} G\right)^{2}+4 \epsilon a_{i}^{+} G a_{i}^{-} G^{-1}\right]
$$

\section{3. $\theta-\phi$ correlator}

We show that reggeization of gluon fails to occur in the effective theory described in the last section, in 2+1 QCD. It seems to us that calculations in [6] concerning Reggeization are also valid in $2+1$ dimensions. Result of this section therefore poses the question whether the ansatz (2.3) need be modified. It remains an open problem whether reggeization occurs in the effective theory in $3+1$ QCD.

One of the reasons for working with $2+1$ QCD is that calculations are extremely simplified, because the effective action is one dimensional and some terms in (2.6) are absent. Moreover, as we shall see in the Sect.5, the color singlet part of (2.1) can be calculated exactly and nonperturbatively with the one dimensional effective action.

If there is only one transverse dimension, "gauge fields" $a^{ \pm}$can always be gauge transformed into zero. The one dimensional effective action is therefore

$$
S[g, h, G]=\int d x \operatorname{tr}\left[\frac{1}{g^{2}} g^{-1} \dot{g} G h^{-1} \dot{h} G^{-1}-i \frac{1}{e^{2}}\left(G^{-1} \dot{G}\right)^{2}\right]
$$

where the dot denotes the derivative with respect to $x$, and $e^{2}=2 g^{2} \log s / \pi$. It is important to notice that so far we have been working in Minkowski spacetime, so there is a factor $i$ in the front of the action in the path integral. The second term in (3.1) is pure imaginary. Together with an overall $i$, it becomes negative in the exponential in the path integral, 
as it should be. From now on we assume that the gauge group is $S U(N)$. To do the perturbative calculation, we expand

$$
g=e^{g \theta}, \quad h=e^{g \phi}, \quad G=e^{e \chi},
$$

The correlator of the two Wilson lines in the amplitude 2.1 is expanded

$$
\langle(g(x)-1)(h(0)-1)\rangle=g^{2}\langle\theta(x) \phi(0)\rangle+\frac{g^{4}}{4}\left\langle\theta^{2}(x) \phi^{2}(0)\right\rangle+\ldots
$$

We are interested in reggeization of gluon which is weighted by a factor $g^{2}$ and a function of $e^{2}$. So this is contained in the first term representing the exchange of the color octet (for $S U(N)$ it is the exchange of adjoint multiplet). To check reggeization, we have to calculate this term at least up to order $e^{4}$. In other words, we have to calculate quantity $\left\langle\theta^{a}(q) \phi^{b}(-q)\right\rangle$ up to two-loops, here we use the notation $\theta=\theta^{a} T_{a}, \phi=\phi^{a} T_{a}$ and the anti-hermitian matrices are normalized by $\operatorname{tr} T_{a} T_{b}=-\delta_{a b}$. For our purpose, it is enough to keep the quadratic term in $\theta-\phi$ in the action (3.1):

$$
S=\int d x \operatorname{tr}\left[\dot{\theta} G \dot{\phi} G^{-1}-\frac{i}{e^{2}}\left(G^{-1} \dot{G}\right)^{2}\right]
$$

At the tree level, the correlator is just the $\theta-\phi$ propagator $-\left(i / q^{2}\right) \delta_{a b}$ in (3.4). At the one-loop level, there are two Feynman diagrams as shown in Fig.1. Solid lines in these diagrams are $\theta-\phi$ propagator, dotted lines are $\chi$ propagator. Only two vertices out of infinite many vertices in the action (3.4) appear in Fig.1. These diagram are easily calculated. The result is

$$
\left\langle\theta^{a}(q) \phi^{b}(-q)\right\rangle=-\frac{i}{q^{2}}\left(1+\frac{N e^{2}}{2} I\right) \delta_{a b},
$$

up to one-loop. The integral $I$ is

$$
I=\int \frac{d k}{2 \pi} \frac{1}{k^{2}+\mu^{2}}
$$

where we have introduced an infrared cut-off $\mu$. The reason for this integral to arise is that all couplings are derivative couplings, as readily seen from (3.4). This integral is different from the standard result [6].

$$
I_{1}=\frac{q^{2}}{2} \int \frac{d k}{2 \pi} \frac{1}{\left(k^{2}+\mu^{2}\right)\left((q-k)^{2}+\mu^{2}\right)}
$$


Note that the usual calculation in $3+1$ QCD is easily transformed into $2+1$ QCD, so the integral (3.7) takes the same form as in [6], except that it is now an one dimensional integral. The difference between $I$ and $I_{1}$ is not crucial when $\mu$ is very small. Both of them can be calculated exactly. $I$ differs from $I_{1}$ by $2 \mu /\left(q^{2}+4 \mu^{2}\right)$, which approaches $\pi \delta(q)$ in the limit $\mu=0$. This difference is zero as long as $q \neq 0$. So we conclude that up to one-loop, the effective action (3.1) yields the same result as in the conventional calculation.

There are seventeen topologically distinct two-loop diagrams. We have drawn fifteen of them in Fig.2. It is seen that at this order, all vertices up to the six vertex as in $2 . \mathrm{h}$ are needed. The sum of diagrams $2 \mathrm{a}-2 \mathrm{k}$ is

$$
-\frac{5 N^{2} e^{4} i}{48 q^{2}} I^{2} \delta_{a b} .
$$

The remaining diagrams in Fig. 2 are obtained from those in Fig.1 by replacing the $\chi$ propagator with its one-loop correction. There are two additional such diagrams not drawn in Fig.2. These are similar to $2 \mathrm{k}$ and $2 \mathrm{~m}$ except that two three vertices attached to the bottom line are replaced by one four vertex. Since $2 \mathrm{l}$ cancels $2 \mathrm{~m}$, the other two also cancel each other. The sum of $2 \mathrm{n}$ and $2 \mathrm{o}$ is

$$
-\frac{N^{2} e^{4} i}{8 q^{2}}\left(I^{2}+\frac{I}{q^{2}} \int \frac{d k}{2 \pi}\right) \delta_{a b} .
$$

The second integral in the parenthesis is ultraviolet divergent. Conventionally this integral is regularized to be zero. We shall see in sect.5 that indeed in an exact calculation of the color singlet part of the correlation of two Wilson lines, there is no ultraviolet divergence. Dropping out this term and taking all results together, the $\theta-\phi$ propagator up to two-loops is given by

$$
\left\langle\theta^{a}(q) \phi^{b}(-q)\right\rangle=-\frac{i}{q^{2}}\left(1-\frac{N e^{2}}{2} I+\frac{11 N^{2} e^{4}}{48} I^{2}\right) \delta_{a b} .
$$

The reggeized gluon propagator is expected to be

$$
-\frac{i}{q^{2}} e^{-\frac{N e^{2}}{2} I} \delta_{a b}
$$

We thus see that result in (3.8) fails to give a reggeized gluon. In the next section, we shall calculate $\theta-\phi$ with by a different method. The result will be the same as in (3.8). That method is very efficient and we do not need to calculate so many diagrams as in Fig.2. Also, the method will be used to do an exact calculation in sect.5. The infrared divergence in $I$ is absent in a color singlet quantity. 


\section{Another calculation of $\theta-\phi$ propagator}

It is technically desirable to develop a simple method to calculate quantities such as $\theta-\phi$ correlator considered in the previous section, to avoid calculating numerous diagrams. In this action we show that the one dimensional action (3.4) can be easily transformed into a simpler action, with which not only perturbative calculation is simplified, but exact result is also available.

The idea is to introduce sources for $\theta$ and $\phi$ and integrate out these fields. To calculate $\theta$ - $\phi$ correlator, we start with action (3.4). Introducing the source term

$$
\int d x \operatorname{tr}\left(\theta J_{\phi}+\phi J_{\theta}\right)
$$

Now the correlator $\left\langle\theta^{a}(x) \phi^{b}(0)\right\rangle$ is written as

$$
-\frac{1}{Z} \frac{\delta^{2} Z}{\delta J_{\phi}^{a}(x) \delta J_{\theta}^{b}(0)},
$$

where the partition function with sources is obtained by integrating out $\theta$ and $\phi$ :

$$
\begin{aligned}
Z= & \int[d G] \operatorname{Det}(G) \exp \left[i \int d z \operatorname { t r } \left(-\frac{i}{e^{2}}\left(G^{-1} \dot{G}\right)^{2}\right.\right. \\
& \left.\left.-\int d x d y \epsilon(z-x) \epsilon(z-y) J_{\phi}(x) G(z) J_{\theta}(y) G^{-1}(z)\right)\right],
\end{aligned}
$$

where the determinant is defined by

$$
\operatorname{Det}(G)=\int[d \theta d \phi] \exp \left(i \int d x \operatorname{tr}\left(\dot{\theta} G \dot{\phi} G^{-1}\right),\right.
$$

and the function $\epsilon(z)$ is the step function $\epsilon(z)=1 / 2$ when $z>0$ and $\epsilon(z)=-1 / 2$ when $z<0$. An infrared cut-off factor $\exp (-\mu|z|)$ is sometimes needed for this function.

It is easy to show diagrammatically that the above determinant gives rise to an ultralocal term in the action, and therefore can be absorbed into the definition of the measure $[d G]$. Hereafter we simply ignore this factor. By a simple manipulation, we find that the correlator is given by

$$
\left\langle\theta^{a}(q) \phi^{b}(-q)\right\rangle=\frac{i}{q^{2}}\left\langle\operatorname{tr}\left(T_{a} G T_{b} G^{-1}\right)(x)\right\rangle .
$$

The expectation value of $\operatorname{tr}\left(T_{a} G T_{b} G^{-1}\right)$ is independent of $x$ and is defined with an action

$$
S(G)=-\frac{i}{e^{2}} \int d x \operatorname{tr}\left(G^{-1} \dot{G}\right)^{2} .
$$


This action is the one dimensional analogue of the principle chiral model. Interestingly, it can be derived from the two dimensional Euclidean QCD on a cylinder, where $G$ is the holonomy around the spatial circle and $x$ is the Euclidean time. However, there is an important difference between the 2D pure QCD and our model, i.e., in the 2D QCD a project operator projecting out color non-singlet wave functions is inserted in the path integral, while there is no such restriction in the model under consideration. Nevertheless, our model can be solved exactly too. Let us calculate (4.2) perturbatively. Since we know that $\left\langle\theta^{a}(q) \phi^{b}(-q)\right\rangle$ is proportional to $\delta_{a b}$, the identity in $(4.2)$ can be further simplified:

$$
\left\langle\theta^{a}(q) \phi^{b}(-q)\right\rangle=\frac{i}{q^{2}} \delta_{a b} \frac{1}{N^{2}-1}\left\langle\sum_{a} T_{a} G T_{a} G^{-1}\right\rangle=-\frac{i}{q^{2}} \delta_{a b} \frac{1}{N^{2}-1}\left\langle\operatorname{tr} G \operatorname{tr} G^{-1}-1\right\rangle .
$$

Expanding $G$ in terms of $\chi$ up to order $e^{4}$, we have

$$
\frac{1}{N^{2}-1}\left\langle\operatorname{tr} G \operatorname{tr} G^{-1}-1\right\rangle=1+\frac{e^{2}}{N^{2}-1}\left\langle\operatorname{tr} \chi^{2}\right\rangle+\frac{e^{4}}{4\left(N^{2}-1\right)}\left\langle\left(\operatorname{tr} \chi^{2}\right)^{2}\right\rangle+\frac{N e^{4}}{12\left(N^{2}-1\right)}\left\langle\operatorname{tr} \chi^{4}\right\rangle
$$

Expectation values on the r.h.s. of the above equation can be easily calculated. We calculate the second term as an example.

$$
\frac{N e^{2}}{N^{2}-1} \frac{1}{\delta(0)} \int \frac{d k}{2 \pi}\left\langle\chi^{a}(k) \chi^{a}(-k)\right\rangle
$$

where the delta function in the denominator comes from the observation that the expectation value $\left\langle\operatorname{tr} \chi^{2}(x)\right\rangle$ is independent of its position, and $\delta(0)$ is just the cut-off of the volume. To the first order, the correlator on the l.h.s. of the above equation is just

$$
\delta(0) \sum_{a} \delta_{a a} \frac{1}{2\left(k^{2}+\mu^{2}\right)}=\delta(0)\left(N^{2}-1\right) \frac{1}{2\left(k^{2}+\mu^{2}\right)}
$$

Plugging this result back into (4.6), we obtain the result $-\left(N e^{2} / 2\right) I$, in agreement with result obtained in the last section. So this simple result obtained from the $\chi$ propagator summarizes two diagrams in Fig.1. Since we are interested in the correlator in (4.4) up to the order $e^{4}$, we have to calculate the next order contribution to (4.6). This is given by the diagram in Fig.3. This single diagram summarizes diagrams 2n and 2o in Fig.2. Plug this quantity into (4.6),

$$
\frac{N e^{2}}{N^{2}-1}\left\langle\operatorname{tr} \chi^{2}\right\rangle=-\frac{N e^{2}}{2} I+\frac{N^{2} e^{4}}{8} I^{2} .
$$


We find that, had there been not for the last two terms in (4.5), this formula would give us reggeization. The last two terms can be calculated similarly. Up to the order $e^{4}$, they are

$$
\begin{aligned}
& \frac{e^{4}}{4\left(N^{2}-1\right)}\left\langle\left(\operatorname{tr} \chi^{2}\right)^{2}\right\rangle=\frac{\left(N^{2}+1\right) e^{4}}{16} I^{2}, \\
& \frac{N e^{4}}{12\left(N^{2}-1\right)}\left\langle\operatorname{tr} \chi^{4}\right\rangle=\frac{\left(2 N^{2}-3\right) e^{4}}{48} I^{2} .
\end{aligned}
$$

These two terms together summarize diagrams 2a-2k. Taking results obtained into (4.5)

$$
\frac{1}{N^{2}-1}\left\langle\operatorname{tr} G \operatorname{tr} G^{-1}-1\right\rangle=1-\frac{N e^{2}}{2} I+\frac{11 N^{2} e^{4}}{48} I^{2}+\ldots
$$

This calculation is in full agreement with (3.8), which was obtained by tediously counting many diagrams.

\section{The color singlet correlator: exact result}

Although we have not seen reggeization of gluon, this does not imply that Verlindes' approach is wrong. What we really have to calculate is color singlet amplitude, and this amplitude will give rise to the hadron scattering amplitude when properly folded by hadron wave functions [12]. From (3.3), the proper quantity to consider is

$$
\frac{g^{4}}{4}\left\langle\operatorname{tr} \theta^{2}(x) \operatorname{tr} \phi^{2}(0)\right\rangle
$$

This is the leading term in the expansion (3.3) when we consider only color singlet combination. High order terms in (3.3) should be included in principle, but it is inconsistent to do this with action (3.4) or even the unsimplified action (2.2), because higher order terms in $g^{2}$ in the action are omitted. However, (5.1) contains all orders in $e^{2}$.

We shall calculate color singlet correlator (5.1) exactly with the action (3.4) in this section, this will constitute our main result in this paper. To do the exact calculation, we introduce sources as in (4.1), then the correlator (5.1) is written as

$$
\left\langle\operatorname{tr} \theta^{2}(x) \operatorname{tr} \phi^{2}(0)\right\rangle=\frac{1}{Z} \frac{\delta^{4} Z}{\delta J_{\phi}^{a}(x) \delta J_{\phi}^{a}(x) \delta J_{\theta}^{b}(0) \delta J_{\theta}^{b}(0)},
$$

and this formula leads to, after integration over $\theta$ and $\phi$

$$
\left\langle\operatorname{tr} \theta^{2}(x) \operatorname{tr} \phi^{2}(0)\right\rangle=-2 \int d z d z^{\prime} \epsilon(z-x) \epsilon\left(z^{\prime}-x\right) \epsilon(z) \epsilon\left(z^{\prime}\right)\left\langle G^{a b}(z) G^{a b}\left(z^{\prime}\right)\right\rangle,
$$


where $G^{a b}=\operatorname{tr}\left(T_{a} G T_{b} G^{-1}\right)$. Using some identities for unitary matrices, the above expression can be further simplified:

$$
\begin{aligned}
\left\langle\operatorname{tr} \theta^{2}(x) \operatorname{tr} \phi^{2}(0)\right\rangle= & -2 \int d z d z^{\prime} \epsilon(z-x) \epsilon\left(z^{\prime}-x\right) \epsilon(z) \epsilon\left(z^{\prime}\right) \\
& \left\langle\operatorname{tr}\left(G^{-1}\left(z^{\prime}\right) G(z)\right) \operatorname{tr}\left(G^{-1}(z) G\left(z^{\prime}\right)\right)-1\right\rangle .
\end{aligned}
$$

Again, the correlator on the r.h.s. of the above equation should be defined with action (4.3). Before we set off to calculate this correlator, we remark that calculation of a general correlator, such as the one discussed in the last section, is very involved, although the particular correlator in (5.2) is surprisingly easy to calculate.

By translational invariance, we set $z^{\prime}=0$. We also assume $z>0$, because the correlator is an even function of $z$. We discretize the $z$ line, the Euclidean action (after a factor $i$ in the path integral is absorbed into the action) is

$$
S(G)=-\frac{1}{e^{2} \Delta z} \sum_{n} \operatorname{tr}\left(G_{n}^{-1} G_{n+1}-1\right)^{2},
$$

where $\Delta z$ is the spacing between two adjacent sites. Define new variable $U_{n}=G_{n}^{-1} G_{n+1}$, then $\operatorname{tr}\left(G^{-1}(0) G(z)\right)=\operatorname{tr}\left(U_{1} \ldots U_{L}\right)$ and $\operatorname{tr}\left(G^{-1}(z) G(0)\right)=\operatorname{tr}\left(U_{L}^{+} \ldots U_{1}^{+}\right)$. Action (5.3) becomes ultra-local in terms of new variables $U_{n}$, this is why the correlator is easy to calculate. It is easy to see that we need only to calculate

$$
\int U_{i j} U_{l k}^{+} \exp \left(\frac{1}{e^{2} \Delta z} \operatorname{tr}(U-1)^{2}\right) d U
$$

Once this quantity is known, we replace $U$ by $U_{N}$ and insert it into the correlator we want to calculate and obtain a recursion relation. Since spacing $\Delta z$ can be arbitrarily small, it is sufficient to calculate quantity (5.4) up to the first order in $e^{2} \Delta z$. This can be readily done and we only give the final result:

$$
\int U_{i j} U_{l k}^{+} \exp \left(\frac{1}{e^{2} \Delta z} \operatorname{tr}(U-1)^{2}\right) d U=\left(1-\frac{N e^{2}}{2} \Delta z\right) \delta_{i j} \delta_{l k}+\frac{e^{2}}{2} \Delta z \delta_{i k} \delta_{j l}
$$

where we assume that the integral $\int \exp \left(\frac{1}{e^{2} \Delta z} \operatorname{tr}(U-1)^{2}\right) d U$ is normalized to one. Defining

$$
F(z)=\left\langle\operatorname{tr}\left(U_{1} \ldots U_{L}\right) \operatorname{tr}\left(U_{L}^{+} \ldots U_{1}^{+}\right)\right\rangle
$$

and inserting into it (5.5) with $U=U_{L}$, we obtain $F(z)=\left(1-\frac{N e^{2}}{2} \Delta z\right) F(z-\Delta z)+\frac{N e^{2}}{2} \Delta z$ or the differential equation

$$
\frac{d F(z)}{d z}=-\frac{N e^{2}}{2} F(z)+\frac{N e^{2}}{2}
$$


The general solution to this equation is $F(z)=1+C \exp \left(-\frac{N e^{2}}{2} z\right), z>0$. To fix the coefficient, let $z=0$, from the definition of $F(z)$ we know that $F(0)=N^{2}$. Thus $C=$ $N^{2}-1$ and $F(z)=1+\left(N^{2}-1\right) \exp \left(-\frac{N e^{2}}{2}|z|\right)$, here $z$ can be either positive or negative. Substituting this exact result into (5.2), we obtain our main result,

$$
\left\langle\operatorname{tr} \theta^{2}(x) \operatorname{tr} \phi^{2}(0)\right\rangle=-2\left(N^{2}-1\right) \int d z d z^{\prime} \epsilon(z-x) \epsilon\left(z^{\prime}-x\right) \epsilon(z) \epsilon\left(z^{\prime}\right) \exp \left(-\frac{N e^{2}}{2}\left|z-z^{\prime}\right|\right)
$$

The rest of this section is devoted to a discussion and comparison of the above exact result to perturbative consideration. Physical implications of this result will be discussed in the next section.

First transforming (5.6) into momentum space, using $\epsilon(k)=\int d x \exp (-i x k) \epsilon(x)=$ $-i k /\left(k^{2}+\mu^{2}\right), \mu$ is a infrared cut-off parameter, we obtain

$$
\begin{aligned}
& \left\langle\operatorname{tr} \theta^{2}(q) \operatorname{tr} \phi^{2}(-q)\right\rangle=-2\left(N^{2}-1\right) \int \frac{d k_{1} d k_{2}}{(2 \pi)^{2}} \epsilon\left(k_{1}\right) \epsilon\left(q-k_{1}\right) \epsilon\left(k_{2}\right) \epsilon\left(q-k_{2}\right) \\
& \int d z \exp \left(i\left(k_{1}-k_{2}\right) z-\frac{N e^{2}}{2}|z|\right) \\
& =-2\left(N^{2}-1\right) \int \frac{d k_{1} d k_{2}}{(2 \pi)^{2}} \epsilon\left(k_{1}\right) \epsilon\left(q-k_{1}\right) \epsilon\left(k_{2}\right) \epsilon\left(q-k_{2}\right) \frac{N e^{2}}{\left(k_{1}-k_{2}\right)^{2}+N^{2} e^{4} / 4} .
\end{aligned}
$$

We now see how the above exact result reproduces perturbative result in the first two orders. Let $e^{2}=0$ in the first equality in (5.7), integration over $z$ yields a delta function $\delta\left(k_{1}-k_{2}\right)$. So to the first order, the correlator is

$$
\left\langle\operatorname{tr} \theta^{2}(q) \operatorname{tr} \phi^{2}(-q)\right\rangle=-2\left(N^{2}-1\right) \int \frac{d k}{2 \pi} \frac{1}{\left(k^{2}+\mu^{2}\right)\left((q-k)^{2}+\mu^{2}\right)} .
$$

This result is the same as given by diagram in Fig.4a. The factor $N^{2}-1$ come from taking trace, and the factor 2 is a symmetric factor. The above integral is infrared divergent, although the full result in (5.7) is finite. Take the next order, and do the integral

$$
\int d z|z| e^{i\left(k_{1}-k_{2}\right) z}=\frac{2\left(\mu^{2}-\left(k_{1}-k_{2}\right)^{2}\right)}{\left(\left(k_{1}-k_{2}\right)^{2}+\mu^{2}\right)^{2}}
$$

where we introduced a cut-off factor in the integration. Because of the infrared singularity in the above formula, we can not simply drop out the $\mu^{2}$ term in the numerator. Plugging the above formula into the first equality in (5.7), it is easy to see, after some simple calculation, that the next order contribution to the correlator is exactly the same as coming from the H-diagram in Fig.4b and two loop corrections to Fig.4a. The H-diagram summarizes 
many contributions in conventional calculation, and the result is written in terms of Lipatov's emission vertex [7]. We thus verified that our exact result agrees with perturbative calculation.

The integral in the second equality of (5.7) is like a generalization of the H-diagram in Fig.4a, with an effective massive $G$-propagator. The mass is $m=N e^{2} / 2$. Note that in $2+1$ dimensions the coupling constant $g^{2}$, therefore $e^{2}$ indeed has a mass dimension. Here physics is drastically different from that in $3+1$ dimensions, where there could be no such mass arising, since the coupling constant there is dimensionless. Finally, we perform the integral in (5.7) and obtain

$$
\left\langle\operatorname{tr} \theta^{2}(q) \operatorname{tr} \phi^{2}(-q)\right\rangle=-\frac{2\left(N^{2}-1\right)}{m\left(q^{2}+m^{2}\right)}+\left(N^{2}-1\right)\left(-\frac{\pi^{2}}{m} \delta^{2}(q)+\frac{2 \pi}{m^{2}} \delta(q)\right) .
$$

The first term in the above formula tells us that the color singlet correlator is effectively due to exchange of a single massive "pomeron". Indeed, combining this result with a factor $g^{4} / 4$ as in formula (5.1), we find that the coupling constant between quark and the pomeron is $g^{2} / \sqrt{2 m}=g \sqrt{\frac{\pi}{2 N \log s}}$. Since this coupling constant depends on the total energy, the "pomeron" has no simple $2+1$ spacetime interpretation. The second term in (5.8) unvanishes only when $q=0$.

\section{Discussion}

\subsection{Hadron-hadron scattering in $2+1 Q C D$}

Upon using wave functions of hadrons, and quark-quark and quark-anti-quark scattering amplitudes, amplitude and cross section of hadron-hadron scattering can be calculated. We calculated in the last section the color singlet part of quark-quark scattering amplitude. It remains to be done the quark-anti-quark scattering amplitude. We now show that as for its color singlet part is concerned, the result is the same as for quark-quark scattering amplitude. According to [2], quark-anti-quark scattering amplitude reduces at high energy to a similar formula as in (2.1), except that $V_{-}$, the Wilson line for the right-moving quark, is replaced by $V_{-}^{*}$ for the right-moving anti-quark. This Wilson line is just $h^{*}$. So the color singlet part of quark-anti-quark scattering amplitude is given by

$$
A(s, t)=\frac{i s}{2 m_{q}^{2}} \frac{g^{4}}{4} \int d z e^{-i q z}\left\langle\operatorname{tr} \theta^{2}(z) \operatorname{tr}\left(\phi^{*}\right)^{2}\right\rangle
$$


Since $\phi$ is an anti-hermitian matrix, $\operatorname{tr}\left(\phi^{*}\right)^{2}=\operatorname{tr}\left(\phi^{t}\right)^{2}, \phi^{t}$ is the transpose of $\phi$. By definition of trace, $\operatorname{tr}\left(\phi^{t}\right)^{2}=\operatorname{tr} \phi^{2}$. Thus, the quark-anti-quark scattering amplitude is identical to quark-quark scattering amplitude, if we are interested only in color singlet.

Consider now hadron-hadron scattering amplitude. Since hadrons are color singlet states, physical processes only involve exchange of color singlet objects. The simplest possible exchange is two gluons, and there are also many corrections to this process. What we calculated in the last section is just these corrections up to all orders in $e^{2}$. Let us calculate elastic scattering amplitude of hadron $A$ and hadron $B$. Assume that the effective vertex for hadron $A$ to emit two virtual gluons is $\Phi_{A}^{\mu_{1} \mu_{2}}\left(k_{1}^{2},\left(q-k_{1}\right)^{2}, s_{1}\right) \delta_{a b}$, where $s_{1}=-2 k_{1} \cdot p_{A}$; and the effective vertex for hadron $B$ is $\Phi_{B}^{\nu_{1} \nu_{2}}\left(k_{2}^{2},\left(q-k_{2}\right)^{2}, s_{2}\right) \delta_{c d}$, where $s_{2}=2 k_{2} \cdot p_{B}$. The scattering amplitude is

$$
\begin{gathered}
A(s, t)=\int \frac{d^{3} k_{1} d^{3} k_{2}}{(2 \pi)^{6}} \Phi_{A}^{\mu_{1} \mu_{2}}\left(k_{1}^{2},\left(q-k_{1}\right)^{2}, s_{1}\right) \Phi_{B}^{\nu_{1} \nu_{2}}\left(k_{2}^{2},\left(q-k_{2}\right)^{2}, s_{2}\right)\left(2 \frac{p_{B}^{\mu_{1}} p_{A}^{\nu_{1}}}{s}\right)\left(2 \frac{p_{B}^{\mu_{2}} p_{A}^{\nu_{2}}}{s}\right) \\
A_{a a, b b}\left(k_{1}, q-k_{1}, k_{2}, q-k_{2}\right),
\end{gathered}
$$

where $A_{a b, c d}$ is the amplitude generalizing two gluon propagators. Following [12], we perform the integral over longitudinal momenta, the resulting integrand depends only on transverse momenta. We define the following "structure functions" depending only on transverse momenta:

$$
\begin{aligned}
\phi_{A}(k, q) & =\int_{L} \frac{d s_{1}}{2 \pi i} \frac{p_{B}^{\mu} p_{B}^{\nu}}{s^{2}} \Phi_{A}^{\mu \nu}\left(k^{2},(q-k)^{2}, s_{1}\right), \\
\phi_{B}(k, q) & =\int_{L} \frac{d s_{2}}{2 \pi i} \frac{p_{A}^{\mu} p_{A}^{\nu}}{s^{2}} \Phi_{B}^{\mu \nu}\left(k^{2},(q-k)^{2}, s_{2}\right) .
\end{aligned}
$$

Contours are chosen to avoid the right and left cuts on the real axis. For details please consult [12]. The hadron-hadron amplitude is

$$
A(s, t)=2 i s \int \frac{d k_{1} d k_{2}}{(2 \pi)^{2}} \phi_{A}\left(k_{1}, q\right) \phi_{B}\left(k_{2}, q\right) A_{a a, b b}^{\prime}\left(k_{1}, k_{2}, q\right)
$$

where $A_{a a, b b}^{\prime}$ can be read off from the color singlet part of quark-quark amplitude. Applying the same procedure to quark-quark scattering, we would obtain a similar formula as (6.4), now the effective vertex for quark to emit two gluons is proportional to $\left(T_{a} T_{b}\right)_{A B}$ (consider color singlet only). Taking trace over $A, B$, it is just $-\delta_{a b}$. Thus, from (5.7), we find

$$
A_{a a, b b}^{\prime}\left(k_{1}, k_{2}, q\right)=\left(N^{2}-1\right) \epsilon\left(k_{1}\right) \epsilon\left(q-k_{1}\right) \epsilon\left(k_{2}\right) \epsilon\left(q-k_{2}\right) \frac{N e^{2}}{\left(k_{1}-k_{2}\right)^{2}+N^{2} e^{4} / 4} .
$$


This amplitude is real. We have taken another sign in (2.1) into account. Note that in (6.2) only two gluon exchange and its corrections are taken into account, while (6.5) contains more terms, such as exchange of three gluons, see [6]. Nevertheless (6.4) still works in this general case, provided we properly modify $\phi_{A}$ and $\phi_{B}$.

The integral in (6.4) has no divergence at $k_{1}=0$ or $k_{2}=0$, for $\phi_{A}$ or $\phi_{B}$ is equal to the difference of values of a function taking at $k_{1}$ and $k_{1}=0 . \phi_{A}$ is a symmetric function of $k_{1}$ and $q-k_{1}$, so there is no divergence at $k_{1}=q$. There is no divergence in (6.4) at $k_{1}=k_{2}$ either, since summation of all orders cures the infrared problem. When the energy is extremely high, function

$$
\frac{N e^{2}}{\left(k_{1}-k_{2}\right)^{2}+N^{2} e^{4} / 4}
$$

does not depend on $k_{1}-k_{2}$ sensitively for a large range of momenta, so it can be effectively replaced by $4 /\left(N e^{2}\right)$. The amplitude (6.4) is approximated by

$$
A(s, t)=\frac{8 i s\left(N^{2}-1\right)}{N e^{2}} \int \frac{d k_{1} d k_{2}}{(2 \pi)^{2}} \phi_{A}\left(k_{1}, q\right) \phi_{B}\left(k_{2}, q\right) \epsilon\left(k_{1}\right) \epsilon\left(q-k_{1}\right) \epsilon\left(k_{2}\right) \epsilon\left(q-k_{2}\right) .
$$

The double integral effectively factorizes into two independent integrals. The double integral is dimensionless, since each structure function $\phi$ contains a factor $g^{2}$ therefore has a mass dimension. $s$ is measured by a certain mass, thus it is dimensionless. We conclude that the amplitude $A(s, t)$ has a dimension of $1 / e^{2}$, the length dimension, as it should be in $2+1$ dimensions. Apart from a factor $s$, the amplitude decreases as $1 / e^{2} \sim 1 / \log s$ at high energies. This physics is quite different from what happens in $3+1$ dimensions, where one expects that apart from $s$, there is an additional Regge-like factor $s^{\alpha}, \alpha>0$. The absence of this factor in $2+1$ dimensions is reasonable: there is no way to construct a dimensionless parameter $\alpha$ out a dimensionful parameter $g^{2}$.

The amplitude (6.4) can be used to calculate the elastic cross section $\sigma_{e}$. In $2+1$ dimensions, the formula of cross section at high energy is given by

$$
\sigma_{e}=\frac{1}{8 \pi s^{2}} \int d q|A(s, t)|^{2}
$$

where the integral is over the transverse component of the momentum transfer. From the above formula and (6.6), we find that the elastic cross section falls off like $1 / e^{4} \sim 1 /(\log s)^{2}$. The total cross section, by the optical theorem, is given by

$$
\sigma_{t} \sim \frac{1}{s} \operatorname{Im} A(s, t=0)
$$


Thus, the total cross section falls off like $1 / e^{2} \sim 1 / \log s$.

In conclusion, we have shown that the high energy behavior of hadron-hadron scattering in $2+1$ differs drastically from that in $3+1$ dimensions. This is not surprising for a couple of reasons. First, the coupling constant in $2+1$ QCD is dimensionful, so the theory is super-renormalizable. This property is reflected in the one dimensional effective action for high energy scattering, there is simply no ultraviolet divergence. For the same reason, there is an effective mass gap $N e^{2}$ arising in high energy scattering. Second, there is only one transverse dimension in $2+1$ spacetime, so there is no arbitrarily high transverse angular momentum. As a result, one does not expect Regge behavior arises, which is due to exchange of states of high spins. The lesson from the study of this paper is that one should be extremely careful in extending any results obtained in lower dimensions to full four dimensions.

\subsection{Some comments on $3+1 Q C D$}

We have used the trick of integrating out the $\theta$ and $\phi$ fields to solve our problem in $2+1$ QCD. This very same trick can be generalized to $3+1$ QCD, starting with the effective action (2.6). Up to the leading order in $g^{2}$, which is what can be done consistently in Verlindes' approach, action (2.6) becomes

$$
S\left[\theta, \phi, G, a^{ \pm}\right]=\int d^{2} z \operatorname{tr}\left[D_{i}^{+} \theta G D_{i}^{-} \phi G^{-1}-\frac{i}{e^{2}}\left(G^{-1} \partial_{i} G\right)^{2}+\frac{i}{e^{2}} a_{i}^{+} G a_{i}^{-} G^{-1}\right],
$$

where we expanded $g$ and $h$ as in (3.2). To integrate out $\theta$ and $\phi$, we do transformation

$$
\phi \rightarrow G^{-1} \phi G, \quad a_{i}^{-} \rightarrow G^{-1} a_{i}^{-} G+G^{-1} \partial_{i} G
$$

Now the first term in (6.7) becomes $\operatorname{tr}\left(D_{i}^{+} \theta D_{i}^{-} \phi\right)$. Integrating out $\theta$ and $\phi$, we obtain the effective action for $G$ and $a^{ \pm}$

$$
S\left[G, a^{ \pm}\right]=i \operatorname{tr} \log \left[-D_{i}^{+} D_{i}^{-}\right]-\frac{i}{e^{2}} \int d^{2} z \operatorname{tr}\left(\left(G^{-1} \partial_{i} G\right)^{2}-a_{i}^{+}\left(a_{i}^{-}+\partial_{i} G G^{-1}\right)\right)
$$

where the determinant is defined with respect to adjoint fields $\theta$ and $\phi$. Now the Wilson line correlation function $(3.3)$ is to be calculated as a correlator involving $G$ and $a^{ \pm}$with the above effective action.

So far we have not chosen a gauge. On might choose the Landau gauge $\partial_{i} a_{i}^{ \pm}=0$, so $a_{i}^{ \pm}=\epsilon_{i j} \partial_{j} \alpha^{ \pm}$. The effective action (6.8) for $G$ and $\alpha^{ \pm}$can be evaluated. The first 
term in (6.8), the term resulting from integrating out $\theta$ and $\phi$, will be similar to a WessZumino-Witten model. This term, we believe, is responsible for the major difference in physics between $3+1 \mathrm{QCD}$ and 2+1 QCD. When the total energy is extremely high, the second term in (6.8) is much smaller compared to the first term, since $e^{2}$ is large as it contains a factor $\log s$. The first term has no scale and therefore is conformally invariant. We thus expect that the leading contribution to the hadron-hadron scattering contains no mass gap. This agrees qualitatively with conventional leading log approach. However, one can not ignore the second term in (6.8), for it contains the kinetic term for $G$. Therefore, $e^{2}$ thus $\log s$ must enter the calculation of scattering amplitude. We leave a detailed study of the model defined by (6.8) to the future.

Acknowledgments We thank J.R. Cudell, E.M. Levin and H. Verlinde for interesting discussions. This work was supported by DOE contract DE-FG02-91ER40688-Task A. 


\section{References}

[1] H. Verlinde and E. Verlinde, preprint PUPT-1319, revised version.

[2] O. Nachtmann, Ann. Phys. 209 (1991) 436.

[3] I.Ya. Areféva, preprint SMI-5-93, hep-th/9306014; SMI-15-93, hep-th/9311115; S.-J. Rey, talk given at 5th Blois Workshop on Elastic and Diffractive Scattering, hep$\mathrm{ph} / 9308332$.

[4] L.N. Lipatov, Nucl. Phys. B365 (1991) 614; R. Kirschner, hep-th/9311159.

[5] L.N. Lipatov, review in Perturbative QCD, ed. A.H. Mueller (World Scientific, Singapore, 1989), and references therein.

[6] H. Cheng and T.T. Wu, Expanding Protons: Scattering at High Energies, The MIT Press (1987).

[7] E. Kuraev, L.N. Lipatov and V. Fadin, Sov. Phys. JETP 44 (1976) 443; 45 (1977) 199; Ya. Balitski and L.N. Lipatov, Sov. Nucl. Phys. 28 (1978) 822.

[8] L.V. Gribov, E.M. Levin, and M.G. Ryskin, Phys. Rep. 100C (1983) 1; E.M. Levin and M.G. Ryskin, Phys. Rep. 189C (1990) 267.

[9] A. Capella, U. Sukhatme, C-I Tan and Tran T. V., Phys. Lett. B81 (1979) 68; see also: Dual Parton Model, Phys. Rep. 236 (1994) 225.

[10] P. V. Landshoff, Proc. of 3rd Int. Conference on Elastic and Diffractive Scattering, Nucl. Phys. B12 (1990) 397.

[11] E.M. Levin and C-I Tan, Heterotic Pomeron: High Energy Hadronic Collisions in QCD, in preparation; a brief outline can be found in Proc. of XXII Int. Symposium on Multiparticle Dynamics, World Scientific (1992).

[12] for example, see L.N. Lipatov, in Perturbative Quantum Chromodynamics, ed. A.H. Mueller, World Scientific, 1989; and E.M. Levin, Orsay lectures on low x deep inelastic scattering, 1991. 


\section{Figure captions}

Fig.1 One-loop diagrams contributing to $\theta-\phi$ correlator.

Fig. 2 Two-loop diagrams contributing to $\theta-\phi$ correlator.

Fig.3 One-loop correction to the $\chi$ propagator.

Fig.4 The box diagram fig.4a and the H-diagram fig.4b, contributing to the color singlet part of quark-quark scattering at the first order and the second order, respectively. 
This figure "fig1-1.png" is available in "png" format from: http://arxiv.org/ps/hep-th/9401134v2 
This figure "fig1-2.png" is available in "png" format from: http://arxiv.org/ps/hep-th/9401134v2 